\title{
Caracterización, MODElado Y ANÁlisis DE FACTIBILIDAD TÉCNICA DE UN SISTEMA DE ACONDICIONAMIENTO DE AIRE POR ADSORCIÓN, UTILIZANDO COLECTORES SOLARES TÉRMICOS COMO FUENTE ENERGÉTICA PRINCIPAL
}

\author{
CHARACTERIZATION, MODELLING AND \\ TECHNICAL FEASIBILITY ANALYSIS OF AN \\ ADSORPTION COOLING SYSTEM USING SOLAR \\ THERMAL COLLECTORS AS THE PRINCIPAL \\ ENERGY SOURCE
}

\author{
Itamar Harris $^{1, *}$, Julio Rodríguez ${ }^{2}$
}

\section{Resumen}

En este artículo se presenta el desarrollo de un trabajo de investigación teórico-experimental de caracterización, modelado y análisis de factibilidad técnica de los sistemas de acondicionamiento de aire por adsorción con fuente de energía solar, utilizando el sistema instalado en el Centro de Innovación Eléctrica, Mecánica y de la Industria (CINEMI) de la Universidad Tecnológica de Panamá. El estudio parte del análisis del sistema de adsorción, su caracterización y modelado matemático, el cual permitió la obtención de expresiones útiles para evaluar parámetros tales como el COP, SCP y la eficiencia térmica del mismo.

\section{Abstract}

This article presents the development of a theoreticalexperimental investigation work which consisted in the characterization, modelling and feasibility analysis of the adsorption cooling systems with solar energy source, using the installed system in the Industry, Mechanical and Electrical Innovation Center (CINEMI), at the Technological University of Panama. The study began with the analysis of the adsorption system, its characterization and the mathematical modelling, which allowed us to obtain useful expressions in order to evaluate some parameters, such as COP, SCP and thermal efficiency of this system.

\footnotetext{
1,*Facultad de Ingeniería Mecánica, Universidad Tecnológica de Panamá - Panamá. Autor para correspondencia : itamar.harris@utp.ac.pa

${ }^{2}$ Facultad de Ingeniería Mecánica, Universidad Tecnológica de Panamá - Panamá.

Recibido: 27-09-2016, aprobado tras revisión: 31-10-2016

Forma sugerida de citación: Harris, I.; Rodríguez, J. (2017). «Caracterización, modelado y análisis de factibilidad técnica de un sistema de acondicionamiento de aire por adsorción, utilizando colectores solares térmicos como fuente energética principal». IngEnIUs. N. ${ }^{\circ} 17$, (Enero-Junio). pp. 5-14. ISSN: 1390-650X.
} 
Utilizando data experimental recabada, se obtuvieron curvas de interés las cuales fueron comparadas con curvas obtenidas mediante simulación numérica. Se concluye que los sistemas de adsorción pueden ser técnicamente viables bajo ciertas condiciones de operación e instalación controlables y ha de tomarse en cuenta las perspectivas para el futuro de esta tecnología y actuales aplicaciones exitosas a nivel mundial.

Palabras clave: acondicionamiento de aire solar, adsorción, colectores solares térmicos.
Using the acquired experimental data it was obtained different curves of interest, which were compared with curves obtained through numerical simulation. It was concluded that the adsorption air-conditioning systems could be technically feasible under some controllable operation and installation conditions; and it is necessary to consider the future perspectives of this technology and the currently successful applications in the world.

Keywords: solar air-conditioning, adsorption, solar thermal collectors. 


\section{Introducción}

La creciente necesidad de acondicionamiento de aire y refrigeración propia de áreas con clima tropical, como el de Panamá, va de la mano con un elevado nivel de radiación solar. Esto ha provocado un especial interés por parte de la comunidad científica, en lograr combinar ambos aspectos para lograr en conjunto un beneficio a las poblaciones [1].

Cabe señalar que el uso de la radiación solar en aplicaciones de enfriamiento de aire es más complejo, tanto a nivel conceptual como en construcción. A pesar de este inconveniente, los esfuerzos relacionados a sus usos siguen en estudio y desarrollo [2].

Bajo el mismo interés de desarrollar e implantar nuevas soluciones tecnológicas fundamentadas en el uso de fuentes renovables de energía, el Centro de Investigación CINEMI realizó el proyecto de Evaluación del uso de colectores solares térmicos en sistemas de absorción para producción de aire acondicionado y/o refrigeración en Panamá [3].

A finales de 2014, tras evaluar sus ventajas para el acondicionamiento con fuente energética solar, se puso en marcha físicamente un sistema de acondicionamiento de aire por adsorción en el complejo universitario de la Universidad Tecnológica de Panamá de Tocumen, en vez de un sistema por absorción como fue considerado en un principio.

La adsorción es un proceso mediante el cual se extrae materia de una fase y se concentra sobre la superficie de otra fase (generalmente sólida); por ello se considera como un fenómeno subsuperficial. La sustancia que se adsorbe se denomina adsorbato y el material sobre el que lo hace es el adsorbente. Al proceso contrario a la adsorción, se le conoce como desorción (o regeneración) [4].

La cantidad de vapor adsorbido por una muestra de un sólido poroso es proporcional a la masa de la muestra y las condiciones experimentales (temperatura y la presión), así como por la naturaleza del sólido (adsorbente) y del vapor (adsorbato). Por lo tanto, la elección de un adsorbente dependerá del vapor a ser adsorbido, además de la capacidad de retención del adsorbente ( $\mathrm{kg}$ adsorbato $/ \mathrm{kg}$ adsorbente) [5].

Los materiales adsorbentes son sales orgánicas e inorgánicas. Sus usos más comunes son la separación de gas-líquido y secado [6]. En nuestro caso particular, el sistema en estudio utiliza sílica-gel como adsorbente.

El sílica-gel o gel de sílice es un sólido fuertemente poroso producido a partir del ácido sulfúrico y el silicato de sodio; el cual está constituido por partículas porosas con diámetros entre 2-20 $\mathrm{nm}$. El sílica-gel tiene una gran capacidad para adsorber vapor de agua (aproximadamente $35-40 \%$ ), con requerimientos de temperaturas relativamente bajas para la regeneración [5].
A principios del siglo XX, entre los años veinte $\mathrm{y}$ treinta se diseñaron y construyeron máquinas de refrigeración por adsorción. En 1925, se registró la primera patente de una máquina de producción de frío, la cual utilizaba como material adsorbente el sílica-gel $\mathrm{SiO}_{2}$ ).

El desarrollo alcanzado respecto al uso de la adsorción para refrigeración, tuvo un estancamiento de aproximadamente cincuenta años; y no es hasta finales de 1970 que los estudios al respecto son retomados; el interés en estos sistemas se incrementó debido a la crisis del petróleo y a los problemas asociados al uso de los compuestos CFC. Es en esta época que se desarrolló un ciclo básico de adsorción utilizando energía solar como fuente energética [7].

A finales del siglo XX, se realizaron nuevos esfuerzos por grupos como Sumathy y Zhongfu (1999), Ferreira y Daguenet (2000) y Hilbrand, Dind, Pons y Buchter (2002), quienes desarrollan sistemas experimentales por adsorción alimentados por energía solar $[8]$.

A nivel comercial, actualmente es posible encontrar en el mercado, máquinas para refrigeración y acondicionamiento de aire por adsorción destinadas a utilizar como fuente energética colectores solares; tanto para aplicaciones comerciales como doméstica.

Para aplicaciones domésticas podemos destacar las desarrolladas por la universidad china SJTU (Shanghai Jiao Tong University), por el fabricante alemán Sortech AG y la del Energy Research Centre of the Netherlands ECN de 2,5 kW [4].

Actualmente se han extendido las áreas de aplicación de las máquinas de adsorción en diversas soluciones tecnológicas para aprovechar las pérdidas de energía, principalmente en forma de calor a la atmósfera, que tienen lugar junto con los procesos conversión de energía. En la actualidad, existen múltiples trabajos que muestran la posibilidad de utilizar chillers de adsorción en sistemas combinados de trigeneración (generación de calor, electricidad y aire acondicionado) [9].

\section{Métodos y materiales}

La máquina de enfriamiento de aire por adsorción (chiller o generador), se compone fundamentalmente de lechos de adsorción ${ }^{1}$, condensador, evaporador y válvula de expansión. Energéticamente, cada lecho es conectado a una fuente de calor a alta temperatura, a una línea de disipación de calor a temperatura intermedia y a una fuente a baja temperatura, donde se produce el efecto útil de producción de frío [7].

$\mathrm{El}$ equipo adquirido funciona de manera continua por lo que dos lechos operan desfasados con el fin de garantizar la continuidad de operación; y el proceso incluye dos periodos de recuperación de calor.

El sistema de acondicionamiento de aire por

\footnotetext{
${ }^{1}$ Cámaras donde se efectúan la absorción y desorción del vapor.
} 
adsorción, instalado en CINEMI (Centro de Investigación e Innovación Eléctrica, Mecánica y de la Industria), ha sido representado esquemáticamente mediante la Figura 1. En la Figura 2 se detalla la simbología utilizada en dicho esquema.

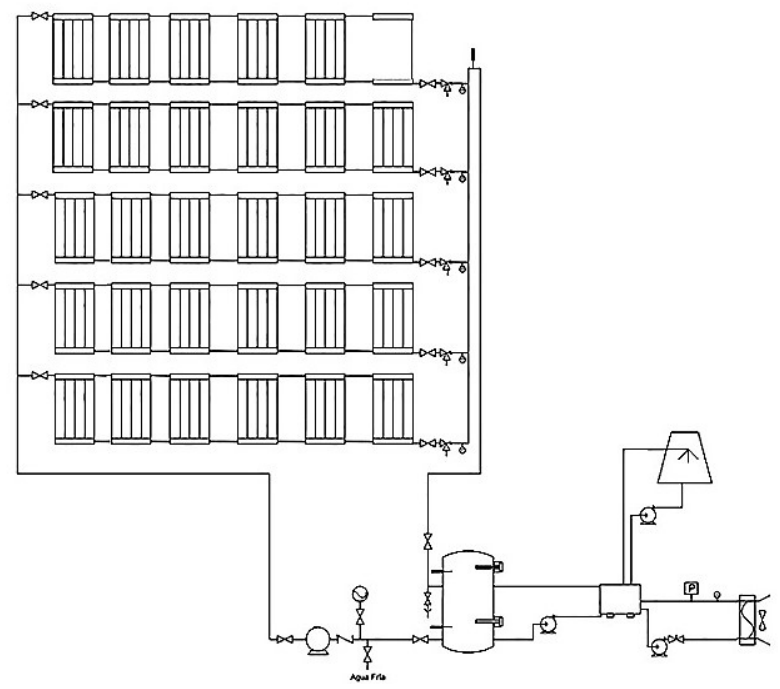

Figura 1. Diagrama del sistema de acondicionamiento de aire por adsorción instalado en CINEMI.

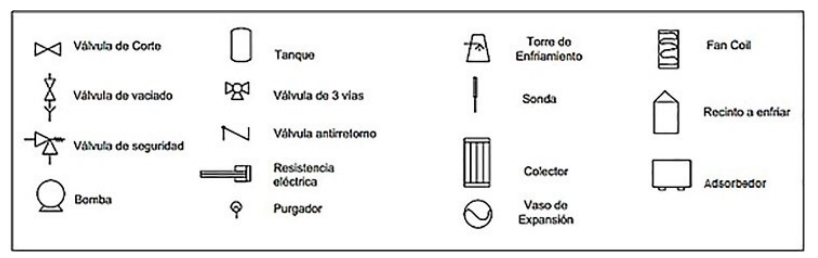

Figura 2. Simbología referente al diagrama del sistema instalado en CINEMI.

Ha de señalarse que este sistema funciona en función de tres circuitos: circuito de agua fría (LT) que es la línea que contiene el fan coil; el circuito de agua caliente (HT) en el cual se encuentra el sistema de colectores; y el circuito de agua de re-enfriamiento a temperatura media (MT) el cual presenta la torre de enfriamiento. Para garantizar el adecuado funcionamiento es necesario que todos los circuitos de agua que fluyen a través del adsorbedor operen a una presión constante de 2 bar.

La máquina de adsorción (o adsorbedor) es, en principio, intermitente: el sólido adsorbe el vapor refrigerante a baja presión y temperatura. Cuando finaliza esta fase, este material se regenera (desorbe) a alta presión y temperatura [7].

Para conseguir un enfriamiento continuo debe disponerse de un par de lechos adsorbente, como mínimo, que realicen los procesos de adsorción/desorción desfasadamente, de modo que mientras en un lecho se adsorbe, en el otro se desorbe vapor.

El ciclo teórico del proceso de adsorción, es representado mediante el diagrama de Clayperon o Dühring (ver Figura 3). Como se puede observar, ese ciclo consta de 4 etapas [10]:

Paso 1: Compresión y calentamiento isostérico ${ }^{2}$ $1 \rightarrow 2$. El adsorbedor en fase de regeneración se aísla del resto de la instalación y se comunica con la fuente de energía calorífica a alta temperatura.

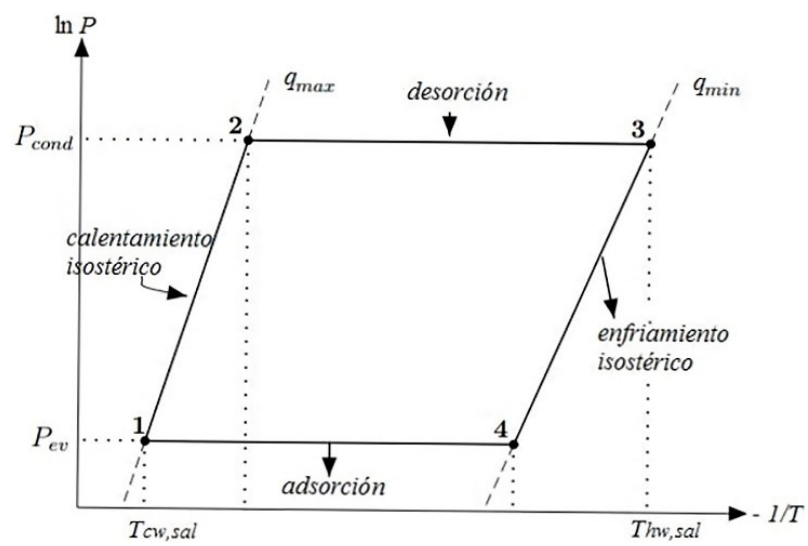

Figura 3. Diagrama de Clapeyron del ciclo básico de adsorción. [10]

Durante el proceso comprendido entre los estados $1 \rightarrow 2$, vapor refrigerante es desorbido isostéricamente.

Paso 2: Desorción a presión constante $2 \rightarrow 3$. En esta fase se sigue calentando el lecho, lo que provoca mayor flujo de vapor desorbido hacia el condensador; hasta que cesa el proceso de desorción cuando el lecho adsorbente alcanza la temperatura de la fuente de calor. Paso 3: Enfriamiento isostérico $3 \rightarrow 4$. El adsorbedor se aísla del resto de la instalación y se enfría mediante el agua de re-enfriamiento. Debido a que se encuentra aislado del resto de la instalación, el adsorbente caliente, al ser enfriado, adsorbe vapor refrigerante (cantidad despreciable) y se suscita una caída de presión y temperatura.

Paso 4: Enfriamiento isostérico $4 \rightarrow 1$. Cuando la presión alcanza la del evaporador, el refrigerante contenido en el evaporador comienza a hervir y luego este fluye hacia el lecho adsorbente, produciéndose el efecto refrigerante.

Es importante destacar que los periodos de recuperación de calor antes mencionados implican el aprovechamiento del calor liberado en la reacción de adsorción (exotérmica), como se muestra en la figura 2. La recuperación de calor de realiza en dos fases. En la fase de recuperación 1 (Fase de R. C. 1), el lecho 1 para calentamiento y lecho 2 para enfriamiento; y en la fase de recuperación 2 (Fase de R. C. 2), el le-

\footnotetext{
${ }^{2} \mathrm{~A}$ concentración de vapor (q) constante.
} 
cho adsorbente 1 para enfriamiento y el lecho 2 para calentamiento (ver Figura 4).
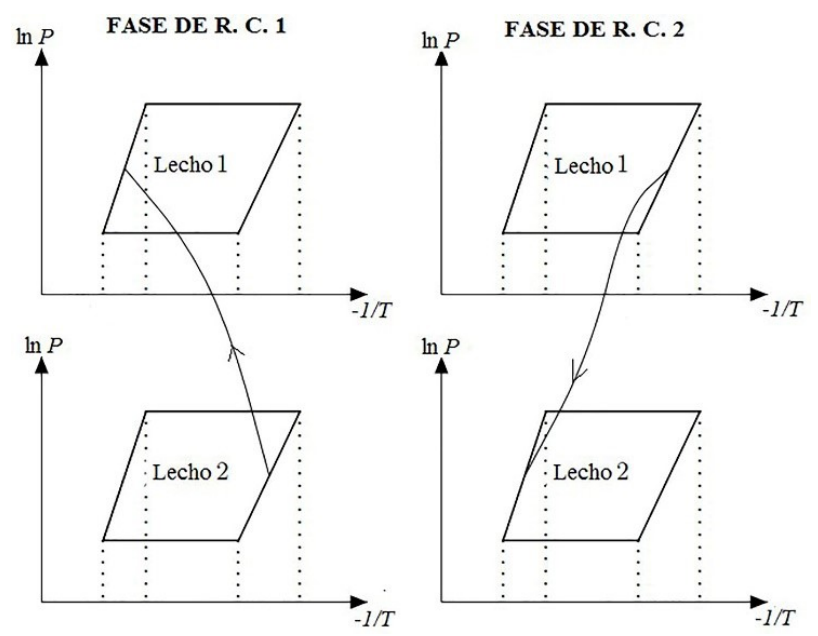

Figura 4. Ciclos de adsorción con dos lechos y recuperación de calor. [10]

Para modelar matemáticamente el funcionamiento del sistema, con respecto a las interacciones energéticas entre cada uno de los circuitos hidráulicos se tomaron en consideración la primera y segunda ley de la termodinámica.

Las suposiciones consideradas para el análisis del sistema en estudio son [11]:

- Temperatura y presión uniformes en los componentes.

- Existe equilibrio termodinámico en el adsorbedor, en cualquier instante de tiempo t.

- La resistencia a la transferencia de masa en el lecho adsorbente es despreciable.

- El calor específico y densidad del adsorbente seco y del agua son constantes.

- La capacidad de enfriamiento del condensador es ilimitada y la temperatura de evaporación es constante.

El propósito de la formulación efectuada, es identificar los parámetros más influyentes y obtener expresiones válidas que permitan evaluar el desempeño del sistema.

Los parámetros de mayor preponderancia en el comportamiento del sistema en estudio son: el coeficiente de desempeño, poder de enfriamiento específico y la eficiencia del sistema.

El coeficiente de desempeño (según sus siglas en inglés, $C O P$ ) de un sistema de acondicionamiento de aire por adsorción puede ser definido en términos de la razón de la energía térmica removida en el proceso de refrigeración $Q_{\text {eva }}$; con respecto a la energía térmica dada al sistema para efectuar dicho proceso $Q_{c h}$. Esto se expresa como,

$$
C O P=\frac{Q_{e v a}}{Q_{c h}}
$$

Siendo

$$
\begin{aligned}
& Q_{e v a}=\dot{m}_{c w} C_{p, c w} \int_{0}^{t_{c i c l o}}\left(T_{c w, e n t}-T_{c w, s a l}\right) d t \\
& Q_{c h} \cong \dot{m}_{h w} C_{p, h w} \int_{0}^{t_{c i c l o}}\left(T_{h w, e n t}-T_{h w, s a l}\right) d t
\end{aligned}
$$

Donde «hw» hace referencia a un parámetro del agua caliente y «cw» a un parámetro del agua fría.

El poder de enfriamiento específico $(S C P$ por sus siglas en inglés), es definido como la tasa a la cual se producefrío con respecto al tiempo de ciclo por unidad de masa de adsorbente. Cuantitativamente se expresa como,

$$
S C P=\frac{\dot{Q}_{e v a}}{m_{S i G}}=\frac{\dot{m}_{c w} C_{p, c w}\left(T_{c w . e n t}-T_{c w, s a l}\right)}{m_{S i G}}
$$

Donde $m_{S i G}$ corresponde a la masa del sílica-gel.

El poder de enfriamiento específico refleja el tamaño del sistema, puesto que se refiere tanto a la masa de adsorbente como a la potencia de refrigeración. Para una carga nominal de enfriamiento, los valores más altos de SCP indican qué tan compacto es el sistema [11].

La eficiencia termodinámica del sistema expresa el desempeño actual del sistema respecto al máximo desempeño esperado de acuerdo con el ciclo ideal de Carnot $\left(C O P_{c t}\right)$. Esta es obtenida mediante la expresión propuesta por Pons [12] como,

$$
\eta_{\text {term }}=\frac{C O P}{C O P_{c t}}
$$

Al realizar las consideraciones pertinentes asociadas con la Segunda Ley de la Termodinámica se obtiene que,

$$
C O P_{c t}=\frac{\frac{T_{\text {prom }}}{T_{g}}-1}{1-\frac{T_{\text {prom }}}{T_{\text {eva }}}}
$$

Donde, si asumimos que las condiciones de equilibrio térmico son alcanzadas

$$
T_{\text {prom }}=\frac{T_{a}+T_{\text {cond }}}{2} \approx T_{a} \approx T_{\text {cond }}
$$

Utilizando las ecuaciones (1), (4) y (5) se obtuvieron diversas curvas que relacionan los parámetros $\mathrm{COP}, \mathrm{SCP}$ y $\eta_{\text {term }}$ con las temperaturas involucradas en dichas expresiones.

Para obtener datos de las temperaturas experimentales, se realizaron pruebas al equipo, en horario de 
11 a. m. a 3 p. m., durante 10 días hábiles. Los datos fueron recabados a intervalos de $30 \mathrm{~s}$.

En este periodo de prueba, se efectúan alrededor de veinte ciclos con cycle times de aproximadamente diez minutos. Dentro de este tiempo de ciclo tienen lugar dos periodos de recuperación de calor, con una duración de $16 \mathrm{~s}$.

\section{Análisis de resultados}

Mediante el manejo de los datos adquiridos, se obtuvieron ciertas curvas de interés la cuales se validaron por comparación con las curvas arrojadas por una simulación numérica mediante aproximación en diferencias finitas, de un sistema de acondicionamiento de aire por adsorción con par sílica-gel-agua con características parecidas; a excepción del hecho de que el mismo, usa la estrategia de recuperación de masa para mejorar la salida de enfriamiento [13].

En la Figura 5 se muestra cómo varía el COP con respecto a la temperatura de la fuente de calor (agua a alta temperatura).

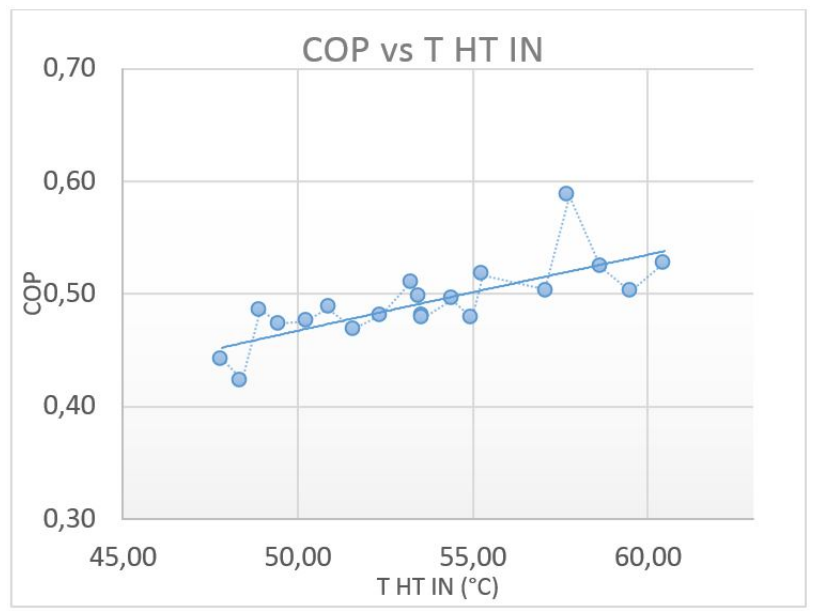

Figura 5. Efecto de la temperatura de la fuente de calor sobre el COP.

Es posible notar que a medida que disminuye dicha temperatura se observa una disminución del COP, lo que coincide con el uso ineficiente de la energía calorífica, puesto que se remueve menor cantidad de calor del recinto para un mismo valor de calor entregado al generador.

A comparar con la simulación (ver Figura 6), solo es posible notar el carácter creciente del COP a medida que aumentaba la temperatura de agua caliente en el rango de temperaturas entre los $45-63^{\circ} \mathrm{C}$.

En cuanto al comportamiento del SCP, vemos que el poder del sílica-gel para enfriar el refrigerante es mayor cuanto mayor sea la temperatura de agua caliente. Se observa que en el rango entre $45-63{ }^{\circ} C$, se da una variación del SCP desde $35 \mathrm{~W} / \mathrm{kg}$ hasta $80 \mathrm{~W} / \mathrm{kg}$, de acuerdo con lo presentado en la Figura 7.

Si consideramos el comportamiento del SCP observado en la Figura 7, para temperaturas entre $65^{\circ} \mathrm{C}$ y $90^{\circ} \mathrm{C}$, se puede esperar un aumento gradual del SCP si la máquina opera con foco caliente en este rango de temperaturas.

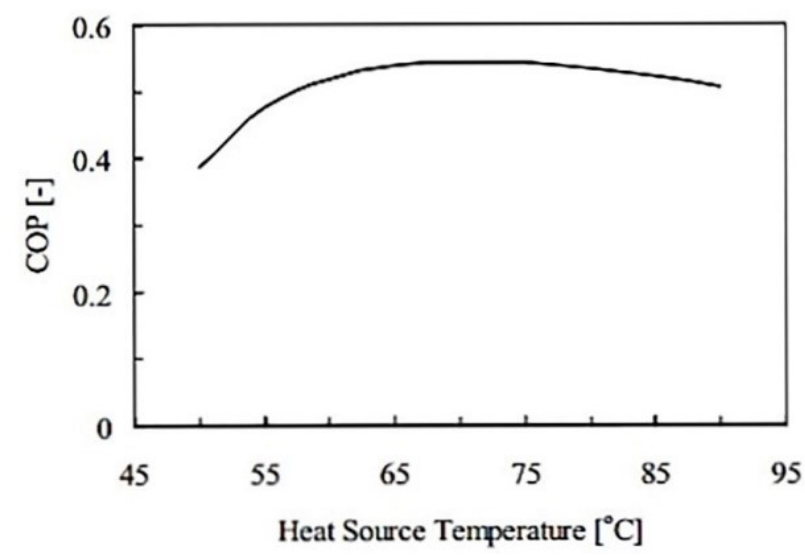

Figura 6. Efecto de la temperatura de la fuente de calor en el COP (obtenido mediante simulación.) [13]

El comportamiento de este parámetro resultó similar al esperado; el cual se muestra en la Figura 8.

De la sección anterior, ya hemos comprobado que la temperatura de agua caliente influye significativamente en el SCP, por lo que podemos decir que también existe una relación directa entre las temperaturas de agua caliente y de agua fría.

En la Figura 9 se relacionan estas temperaturas y los resultados son los esperados: a medida que aumenta la temperatura de agua caliente, disminuye la temperatura de agua fría.

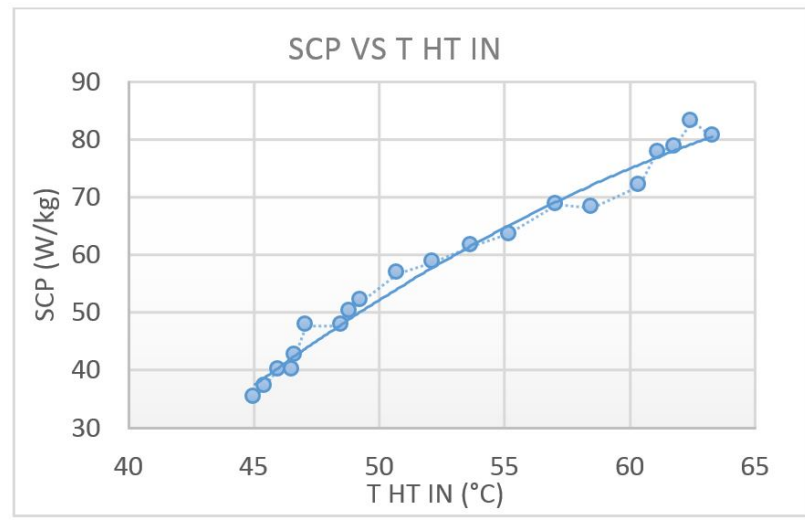

Figura 7. Efecto de la temperatura de la fuente de calor sobre el SCP. 


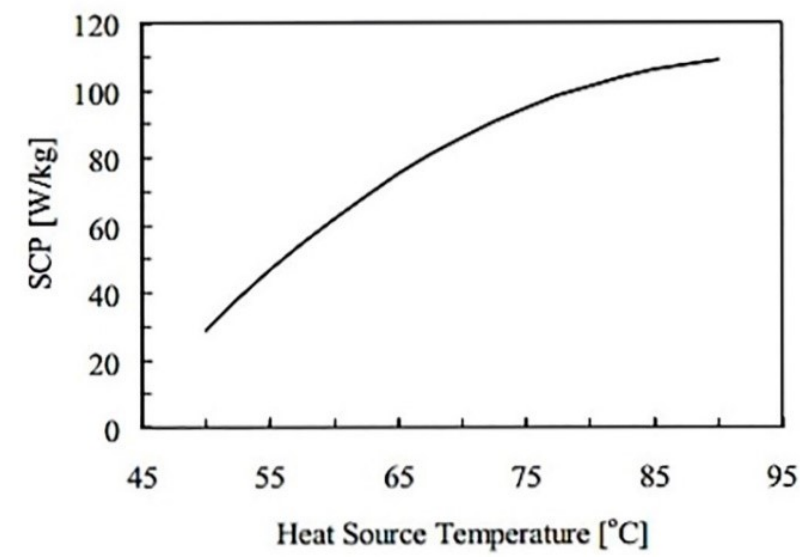

Figura 8. Efecto de la temperatura de agua caliente sobre el SCP (simulación). [13]

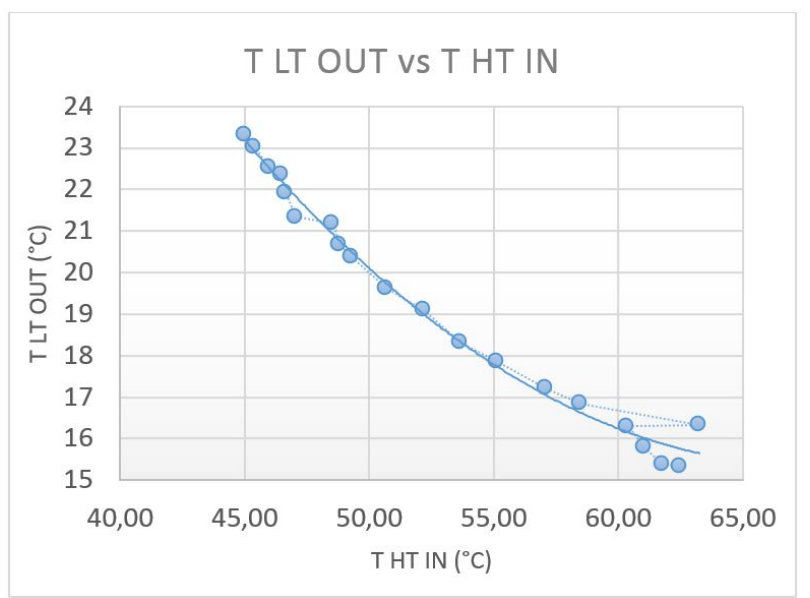

Figura 9. Efecto de la temperatura de agua caliente sobre la de agua fría.

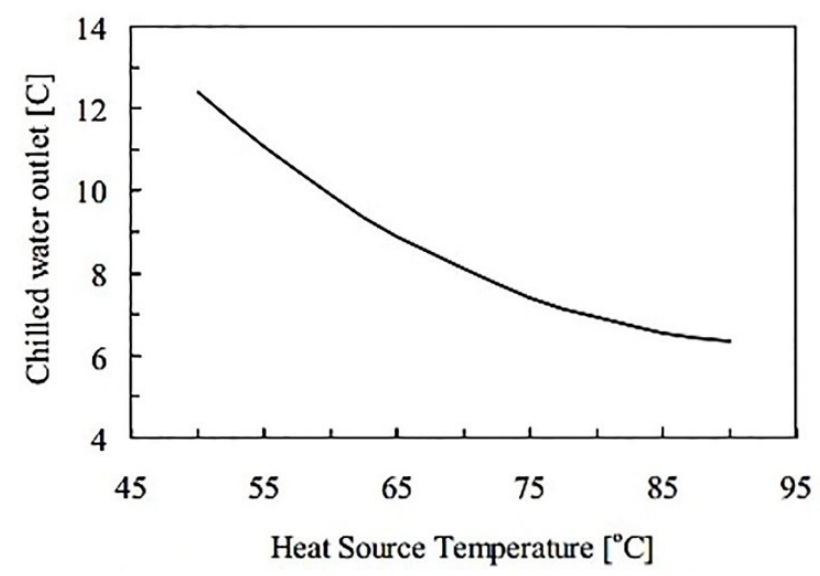

Figura 10. Efecto de la temperatura de agua caliente sobre la de agua fría (simulación). [13]

Sin embargo, atendiendo a los resultados obtenidos en la simulación (ver Figura 10), se esperaba que las temperaturas de agua caliente alcanzadas resultaran en temperaturas de agua fría más bajas.

Cabe destacar que factores como el tipo de sílicagel, el efecto de la estrategia de recuperación de masa asumido en la simulación y las características propias de los intercambiadores de calor son factores que influyen en este resultado.

En cuanto a la relación del SCP con la temperatura T MT IN, en la figura 11 se observa que la curva obtenida tiende a una relación polinómica de segundo orden, con concavidad negativa. Dicho aspecto demuestra una tendencia de gran disminución del SCP con el aumento de la temperatura T MT IN.

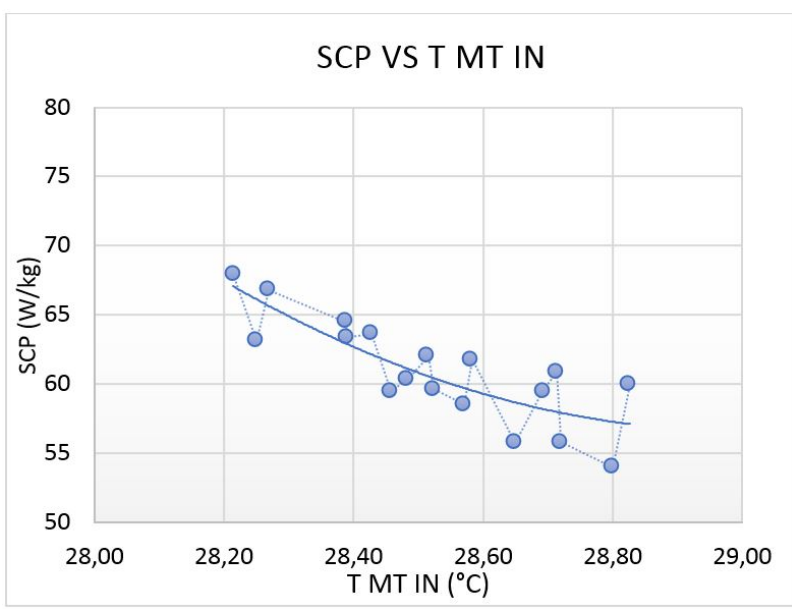

Figura 11. Efecto de la temperatura de agua de reenfriamiento en el comportamiento del SCP.

Si atendemos a la forma de la curva propuesta por la simulación (Figura 12), se esperaba una disminución con concavidad inversa a la obtenida experimentalmente, lo que se traduce en una menor razón de disminución del SCP con el aumento de la temperatura de agua de re-enfriamiento.

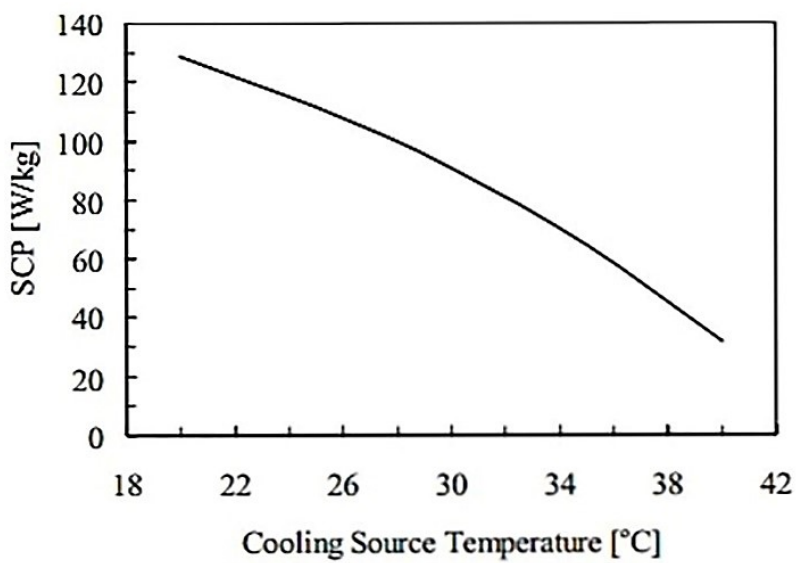

Figura 12. Efecto de la temperatura de agua de reenfriamiento en el comportamiento del SCP (simulación). [13] 
En la Figura 13, nos muestra la relación decreciente de la eficiencia a medida que la temperatura de dicha fuente disminuye.

En esta figura ha de notarse que el máximo valor alcanzado de eficiencia es de un $80 \%$. Se verifica una disminución de aproximadamente $5 \%$ de eficiencia, con la disminución de la temperatura en solo $1^{\circ} \mathrm{C}$.

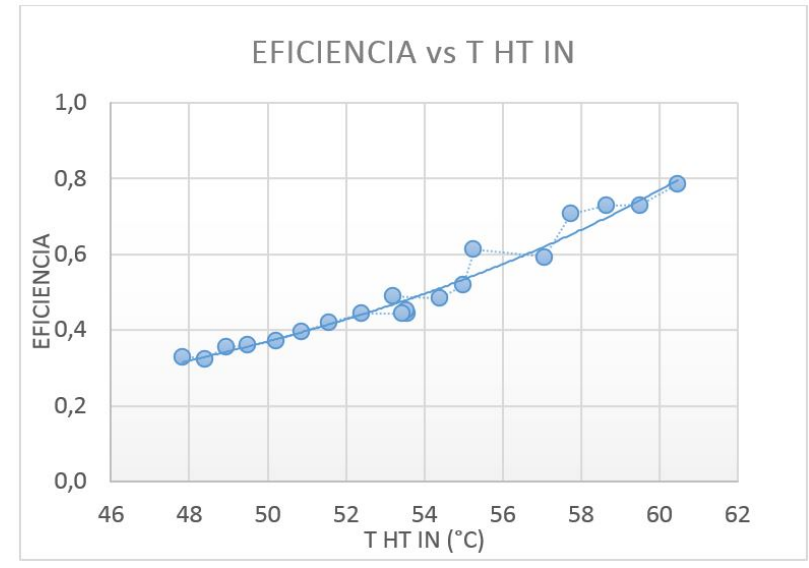

Figura 13. Efecto de la temperatura de agua caliente en la eficiencia del sistema.

Como otro punto relevante, en la Figura 14, se presentan las curvas obtenidas de temperatura de bulbo seco y humedad relativa en un día de prueba.

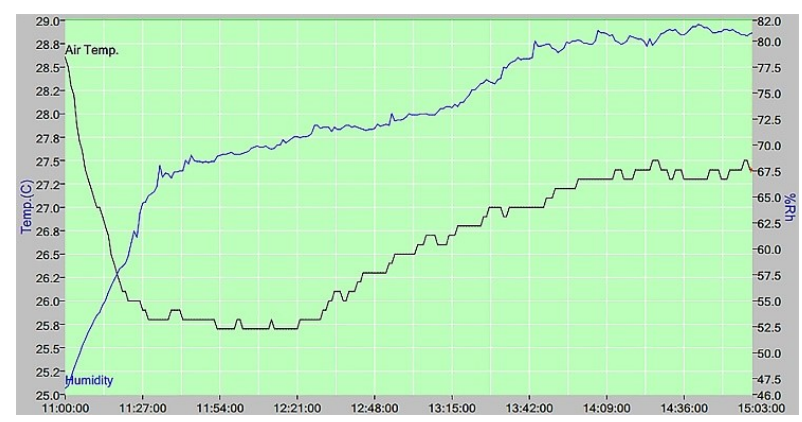

Figura 14. Comportamiento de la temperatura de bulbo seco y humedad relativa del aire del recinto en un periodo de prueba.

Para todos los días de prueba, la disminución del nivel de la radiación solar provoca la disminución de la temperatura de agua caliente por lo que el sistema no logra mantener la temperatura de bulbo seco en el recinto.

Notamos un continuo aumento de la humedad relativa, lo cual es cónsono con el hecho de que se usa un fan coil como elemento para suministrar aire enfriado al recinto, con las características del clima propio de nuestro país y con el hecho de que el sistema no cuenta con algún mecanismo de control de humedad.

Además de realizarse mediciones de temperaturas de operación y condiciones del aire en el recinto, se recabó información respecto al consumo eléctrico del sistema.

Dependiendo de las condiciones climáticas suscitadas durante cada día de prueba, el equipo funcionó bajo dos condiciones de operación: con el apoyo de una fuente de calor auxiliar o sin dicho respaldo.

Cuando el equipo funciona sin el uso de la resistencia eléctrica, su consumo oscila entre 0,946 kWh y 1,073 $k W h$. Al funcionar utilizando la resistencia eléctrica, el consumo oscila entre $3,02 k W h$ y $3,41 k W h$, lo cual se traduce en que la resistencia eléctrica consume entre $2,27 k W h$ y $2,49 k W h$ per sé. Se observa que la utilización de la resistencia eléctrica aumenta el consumo eléctrico del sistema en un $318 \%$; es decir, se triplica.

\section{Análisis de factibilidad}

Se procedió a realizar una comparación de las características operativas del sistema instalado con respecto a las de un split modelo Samsung ASV-24PSBAN de $18000 \mathrm{Btu} / \mathrm{h}$ (equivalente a $5,28 k W_{t}$ ), de igual forma instalado en el recinto de prueba.

Los parámetros comparados (ver tabla 1) fueron consumo eléctrico, temperatura, humedad relativa del aire y dimensiones de elementos internos en el recinto.

Basándonos en la comparación realizada podemos señalar que a nivel técnico, el sistema de acondicionamiento de aire por adsorción implementado puede ser factible, bajo condiciones de funcionamiento e instalación específicas.

Tabla 1. Comparación de algunos parámetros de un sistema Split vs. un sistema de adsorción

\begin{tabular}{lcc}
\hline \multicolumn{1}{c}{ Parámetros } & Split & Sistema de adsorción* \\
\hline Capacidad & $5,28 k W_{t}$ & $5,5 k W_{t}$ \\
Consumo eléctrico & $1,12 k W_{e}$ & $1,07 k W_{e}$ \\
Temperatura & $26{ }^{\circ} \mathrm{C}$ & $26{ }^{\circ} \mathrm{C}$ \\
Humedad relativa & $64 \%$ & $75 \%$ \\
$\begin{array}{l}\text { Dimensiones de } \\
\text { elementos internos en }\end{array}$ & & Generador: \\
el recinto (ancho x & $1,065 \times 0,230 \times 0,298 \mathrm{~m}$ & $0,798 \times 0,821 \times 1,345 \mathrm{~m}$ \\
profundidad x altura) & & Fan coil: \\
* Valores promedio de operación. & & \\
\end{tabular}

Notamos que la capacidad de enfriamiento de ambos dispositivos es semejante y el consumo de energía eléctrica se reduce en aproximadamente un $50 \%$ (si no se hace uso de la resistencia eléctrica).

Sin embargo, en la mayoría de las ocasiones fue necesario el uso de la resistencia eléctrica para suplir la carga, lo que nos indica que el sistema depende del uso de una fuente auxiliar de suministro de energía.

Además, otro parámetro crítico puede ser la humedad relativa, en caso de operar durante largos periodos. Actualmente, no se cuenta con algún dispositivo que permita controlar la humedad relativa en el recinto lo cual, se prevé, ha de provocar condiciones de bajo comfort tras largos periodos de operación. 
En cuanto a este aspecto, es preciso hacer referencia al estudio realizado por Dai et al. (2002) que consistió en el estudio teórico de un sistema híbrido de acondicionamiento de aire por adsorción y por desecante; instalado en un depósito para almacenamiento de granos de la Universidad Shanghai Jiao Tong [14].

La deshumidificación del aire que se realiza mediante el sistema de acondicionamiento con desecante, disminuye la carga de enfriamiento del recinto, lo que se traduce en un mejor desempeño del sistema de acondicionamiento por adsorción [15].

Un punto crítico, no señalado a nivel de la comparación técnica, es la diferencia de costos de los dos sistemas; siendo el de adsorción 49 veces más costoso que el sistema Split de referencia. Sin embargo, dicha diferencia es menos considerable cuando orientamos su aplicación al acondicionamiento de recintos con alta carga de enfriamiento.

Según [10], cuanto mayor es la inversión inicial (lo cual implica el uso de un sistema de mayor capacidad de enfriamiento), menores son los costos de instalación de este tipo de sistemas en comparación con los asociados a la implementación de un sistema por compresión.

En este contexto cabe señalar las perspectivas para el futuro de la tecnología utilizada. Se prevé por Wang et. al (2001) que esta tecnología a nivel residencial tendrá un futuro más prometedor en tanto se logre: desarrollar materiales adsorbentes que adsorban mayores concentraciones de vapor, mejorar de la transferencia de calor entre las partículas adsorbentes y el material del intercambiador de calor, desarrollar sistemas compactos que integren todos sus componentes y trabajar aún más en estrategias de control de bajo costo y alto rendimiento [10].

Dado esto, Otanicar et. al (2012) concluyeron mediante la comparación (a nivel de costos e impacto medioambiental) de sistemas de acondicionamiento solar para aplicación residencial cuyas fuentes energéticas fueron solar térmica (mediante colectores solares) y solar fotovoltaica (mediante paneles fotovoltaicos: PV); que tanto el impacto medioambiental y los costos de instalación son menores para los sistemas con fuente energética fotovoltaica. Se prevé en su estudio que para 2030 los costos asociados a los sistemas PV sean reducidos en un 60-77\% y en el caso de los sistemas con fuente solar térmica en solo un 35-45 \% [16].

Independientemente de las proyecciones de la tecnología de adsorción con fuente de energía solar térmica para el caso de aplicación residencial, es necesario tomar en consideración su aplicabilidad para sectores en los que existen grandes pérdidas de energía térmica.

En este punto encontramos un gran potencial a nivel de sistemas combinados de trigeneración (CCHP, por sus siglas en inglés combinated cooling, heating and power) cuyas eficiencias oscilan entre 70-80\% [17].

\section{Conclusiones}

Se obtuvieron expresiones válidas que permitieron evaluar el desempeño del sistema a partir del modelado matemático realizado y el uso de la data obtenida en las pruebas experimentales. Las curvas obtenidas demuestran un comportamiento estándar del sistema de adsorción, puesto que se nota una alta similitud de las mismas, con respecto a los resultados de las simulaciones presentadas en la referencia consultada.

Fueron identificados factores que señalan que el equipo puede ser viable a nivel técnico, puesto que puede suplir la carga de enfriamiento involucrando un menor consumo de energía eléctrica al no utilizar una resistencia eléctrica, lo que recalca la importancia de optimizar la fuente auxiliar de suministro de energía calorífica y el sistema de colectores.

Sin embargo, a nivel residencial, las posibles ventajas técnicas están condicionadas por la disponibilidad de la fuente energética y el desarrollo de sistemas de acondicionamiento por adsorción que resulten más económicos, compactos; y que permitan manejar de mejor manera la humedad relativa.

Basados en lo descrito previamente, se considera conveniente tomar en consideración las perspectivas en el futuro de los sistemas residenciales de acondicionamiento con fuente energética solar térmica y contemplar la posibilidad de uso a mayor escala, como parte de sistemas combinados de trigeneración (CCHP, por sus siglas en inglés combinated cooling, heating and power) en la realización de futuras investigaciones relacionadas.

\section{Referencias}

[1] V. Mittal, K. Kasana, and N. Thakur, "Modelling and simulation of an absorption cooling system for india," Journal of Energy in Southern Africa, vol. 17 , no. 3, pp. 65-70, 2006.

[2] R. Z. Wang, T. S. Ge, C. J. Chen, Q. Ma, and Z. J. Xiong, "Solar sorption cooling systems for residential applications: Options and guidelines," International Journal of Refrigeration, vol. 1, no. 32, pp. 638-660, 2009.

[3] E. Quintana and R. Díaz, "Simulación de un sistema de acondicionamiento de aire por absorción con asistencia solar en panamá utilizando trnsys," I+D Tecnológico, vol. 9, no. 2, pp. 48-58, 2013.

[4] E. Torrella Alcaraz, D. Sánchez García-Vacas, R. Cabello López, J. Patiño Pérez, and R. Llopis Doménech. (2011) Las máquinas de producción de frío por adsorción con la mezcla sílica-gel-agua. [Online]. Available: http://repositori.uji.es/xmlui/bitstream/ handle/10234/41841/52043.pdf?sequence=2. 
[5] S. T. Oh, "Siligel/water based adsorption cooling system employing compact fi-tube heat. exchanger," Kyushu University, Japan, 2013.

[6] J. G. Trujeque Bolio and G. A. Ramos López, "Diseño de refrigerador solar por adsorción para clima cálido húmedo," Tesis de Posgrado en Tecnología Avanzada, Instituto Politécnico Nacional, Querétaro, pp. 115, 2010.

[7] G. Tashtoush, "A statistical approach to optimize the solar adsorption refrigeration system," CS Canada Energy Science and Technology, vol. 3, no. 2, pp. 18-28, 2012.

[8] W. Hussein, "Solar energy refrigeration by liquidsolid adsorption technique," Master's thesis, Nablus - Palestine: An-Najah University, 2008.

[9] M. Szyc and W. Nowak, "Operation of an adsorption chiller in different cycle time conditions," Chemical Process Engineering, vol. 35, no. 1, pp. 109-119, 2014.

[10] D. E. Demirocak, "Thermodynamic and economic analysis of a solar thermal powered adsorption cooling system," Thesis of Master of Science in Mechanical Engineering, Middle East Technical University, 2008.

[11] L. Y. K. Sumathy, "Modeling and simulation of a solar powered two bed adsorption air condition- ing system," Energy Conversion and Management, vol. 45, pp. 2761-2775, 2004.

[12] E. Anyanwu and N. Ogueke, "Thermodynamic design procedure for solid adsorption solar refrigerator," Renewable Energy, no. 30, pp. 81-96, 2005 .

[13] M. Z. I. Khan, S. Sultana, A. Akisawa, and T. Kashiwagi, "Numerical simulation of advanced adsorption refrigeration chiller with mass recovery," Journal of Naval Architecture and Marine Engineering, vol. 1, no. 3, pp. 59-67, 2006.

[14] H. L. Luo, Y. Dai, and R. Z. Wang, "Experimental investigation of a solar adsorption chiller used for grain depot cooling," Applied Thermal Engineering, no. 26, pp. 1218-1225, 2006.

[15] Y. J. Dai, R. Z. Wang, and Y. X. Xu, "Study of a solar powered solid adsorption - desiccant cooling system used for grain storage," Renewable Energy, no. 25 , pp. 417-430, 2002.

[16] T. Otanicar, R. A. Taylor, and P. E. Phelan, "Prospects of solar cooling - an economic and environmental assessment," Solar Energy, no. 86, pp. 1287-1299, 2012.

[17] D. W. Wu and W. R. Z., "Combined cooling, heating and power: A review," Progress in Energy and Combustion Science, no. 32, pp. 459-495, 2006. 